\title{
Systematic review: the perceptions, diagnosis and management of irritable bowel syndrome in primary care - A Rome Foundation Working Team Report
}

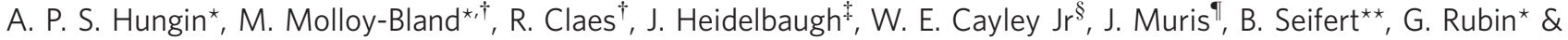 \\ N. de $\mathrm{Wit}^{\dagger \dagger}$
}

*School of Medicine, Pharmacy and

Health, Durham University,

Stockton-on-Tees, UK.

${ }^{\dagger}$ Research Evaluation Unit, Oxford

PharmaGenesis ${ }^{\text {TM }}$ Ltd, Oxford, UK.

*Department of Family Medicine,

University of Michigan, Ann Arbor, MI, USA.

$\S$ Department of Family Medicine, University of Wisconsin, Eau Claire, WI, USA.

"Department of Family Medicine, Maastricht University, Maastricht, The Netherlands.

**Department of General Practice, First Faculty of Medicine, Charles University in Prague, Prague, Czech Republic.

†'Department of General Practice, Julius Center for Health Sciences and Primary Care, University Medical Center Utrecht, Utrecht,

The Netherlands.

\section{Correspondence to:}

Prof. A. P. S. Hungin, School of Medicine, Pharmacy and Health, Wolfson Research Institute, Durham University, Queen's Campus, Stockton-on-Tees TS17 6BH, UK. E-mail: a.p.s.hungin@durham.ac.uk

\section{Publication data}

Submitted 8 April 2014

First decision 29 April 2014

Resubmitted 19 August 2014

Accepted 27 August 2014

EV Pub Online 17 September 2014

This uncommissioned review article was subject to full peer-review.

\section{SUMMARY}

\section{Objective}

To review studies on the perceptions, diagnosis and management of irritable bowel syndrome (IBS) in primary care.

\section{Methods}

Systematic searches of PubMed and Embase.

\section{Results}

Of 746 initial search hits, 29 studies were included. Relatively few primary care physicians were aware of (2-36\%; nine studies) or used $(0-21 \%$; six studies) formal diagnostic criteria for IBS. Nevertheless, most could recognise the key IBS symptoms of abdominal pain, bloating and disturbed defaecation. A minority of primary care physicians [7-32\%; one study (six European countries)] preferred to refer patients to a specialist before making an IBS diagnosis, and few patients [4-23\%; three studies (two European, one US)] were referred to a gastroenterologist by their primary care physician. Most PCPs were unsure about IBS causes and treatment effectiveness, leading to varied therapeutic approaches and broad but frequent use of diagnostic tests. Diagnostic tests, including colon investigations, were more common in older patients $(>45$ years) than in younger patients [ $<45$ years; five studies (four European, one US)].

\section{Conclusions}

There has been much emphasis about the desirability of an initial positive diagnosis of IBS. While it appears most primary care physicians do make a tentative IBS diagnosis from the start, they still tend to use additional testing to confirm it. Although an early, positive diagnosis has advantages in avoiding unnecessary investigations and costs, until formal diagnostic criteria are conclusively shown to sufficiently exclude organic disease, bowel investigations, such as colonoscopy, will continue to be important to primary care physicians. 


\section{A. P. S. Hungin et al.}

\section{INTRODUCTION}

Irritable bowel syndrome (IBS) is a functional bowel disorder that is characterised by abdominal pain, bloating and disturbed defaecation. ${ }^{1}$ IBS affects an estimated 10$15 \%$ of people in Western Europe and North America ${ }^{2-5}$ and $5-10 \%$ in Asia. ${ }^{6}$

Since no specific biological markers for IBS have been identified, clinicians usually rely on symptom-based criteria for diagnosis. A number of diagnostic tools have been developed for use in IBS including the Rome criteria, which were last revised in 2006, ${ }^{7}$ and the Manning criteria. ${ }^{8}$ Diagnostic criteria have also been developed for use in primary care. ${ }^{9}$ The Rome criteria are the most widely accepted among gastroenterologists and are used as research and diagnostic tools. However, according to a recent systematic review, few studies have validated the Rome I or Rome II criteria, ${ }^{10}$ and no consistent differences have been observed in the sensitivity or specificity of the Rome I, Rome II and Manning criteria. ${ }^{11}$ Furthermore no studies have validated Rome III criteria, ${ }^{10}$ and their uptake has been variable in clinical practice, possibly because they were developed partly for research purposes. ${ }^{10}$ There is still a need for development and validation of diagnostic criteria in primary care practice, to address patients' and physicians' concerns that organic disease might be missed without endoscopy.

Treatment strategies for IBS are also based on the nature, type and severity of symptoms. ${ }^{2}$ Although generally speaking the effectiveness of drug treatment in IBS is limited, several treatments have been shown to be superior to placebo. These include anti-spasmodic agents and drugs acting on the 5-hydroxytryptamine receptor for diarrhoea-predominant IBS (IBS-D), soluble fibre for increasing stool-frequency in constipation-predominant IBS (IBS-C), chloride channel agonists for IBS-C and anti-depressants for chronic pain. ${ }^{12}$ In addition, several psychotherapeutic interventions have established effectiveness in IBS. ${ }^{13,}{ }^{14}$ However, there is a need for further consensus and guidance on which treatments should be used for which patients with IBS, as was done recently for probiotics. ${ }^{15}$

Less than half of those suffering from IBS consult a physician. ${ }^{16}$ Although most clinical studies on IBS have been performed in patients referred to gastroenterologists, the majority of patients are likely to present in primary care where their diagnosis and management is initiated. Previous reviews of IBS in primary care have focused on the interactions between PCPs and patients with IBS, ${ }^{17}$ and on differences/similarities in IBS between primary and secondary care. ${ }^{18}$ Here, we aimed to focus on reviewing the literature on PCPs perceptions, understanding and views of IBS, including how they choose to diagnose and manage this challenging problem.

\section{METHODS}

\section{Search strategy}

We employed broad systematic search terms aimed at identifying any studies mentioning IBS and primary care in the title/abstract, or indexed in PubMed under related Mesh terms (Figure 1). PubMed filters were applied to limit identified articles to those conducted in humans and published in English in the last 20 years (up to 10 November 2013). This search strategy was adapted for use in Embase, which was accessed via the online search platform OvidSP. The results were screened by title and abstract to exclude clearly irrelevant articles and those not specifically examining IBS in primary care. Full papers were obtained for the remaining articles to identify those providing insight into PCPs' perceptions and understanding of IBS in primary care, as well as its diagnosis and management in this setting. Owing to the broad nature of the study question, more specific predefined inclusion criteria were not able to be applied. The literature search was supplemented by relevant papers from the authors' own libraries. Screening of the searches, data extraction and selection of the final articles was conducted by a single reviewer (MM-B) and independently verified by a second reviewer (RC). A formal assessment of the quality of the included studies was not conducted for this review.

\section{RESULTS}

\section{Identified studies}

Overall, 29 studies were included (Figure 1 and Table S1). Of these, 20 were conducted in Europe, seven in North America, one in the Middle East and one in Southeast Asia. The publication date ranged from 1997 to 2013 (median: 2006) and all studies collected data using questionnaires, interviews and/or medical chart reviews.

While a formal assessment of study quality was not conducted, it should be noted that response rates for study participation in the included studies were generally low, and often not reported (Table S1). Furthermore, while publication dates are included throughout this article so they can be factored into the interpretation of 


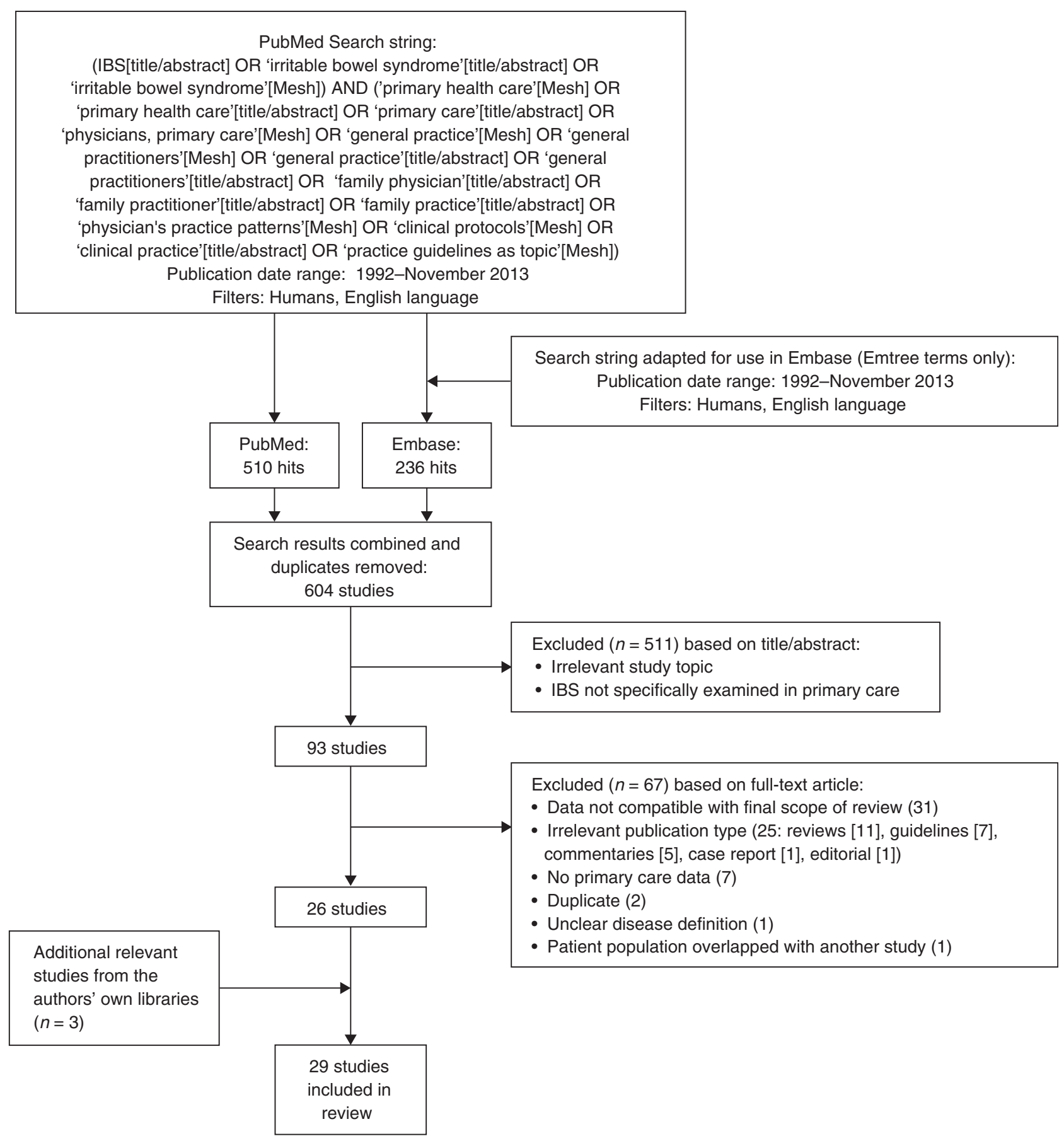

Figure 1 | Flow diagram of literature searches. The PubMed and Embase searches were performed up to 11 Nov 2013, and were limited to those conducted in humans and published in English in the last 20 years.

the data, study periods were rarely reported. Unknown variation in the time between study conduct and publication should therefore be acknowledged. Finally, much of the data presented here pertains to PCPs' perceptions and views of IBS. Such data can only be obtained through surveys, which have obvious limitations in terms of bias. Other data, such as those on the use of diagnostic tests, treatments and referral rates, were collected using methods that vary in terms of their reliability. In general (though not always), data gathered via medical chart review will be less biased than questionnaire data, and prospective questionnaires will be less biased than 


\section{A. P. S. Hungin et al.}

those that are applied retrospectively (due to recall bias). Data sources used are thus described throughout the text, when this may aid in interpreting the reliability of the data.

\section{Use of diagnostic tests}

Most PCPs in the European survey by Seifert et al. (2008) used diagnostic tests for IBS, with $35 \%$ of Dutch PCPs, 25\% of British PCPs and less than 10\% of PCPs in other countries stating that they would not use diagnostic tests for IBS. ${ }^{19}$ These results are consistent with other studies that show around two-thirds of patients with IBS in primary care usually undergo some form of diagnostic testing. ${ }^{20-22}$

Substantial variation exists in the types of diagnostic tests used by PCPs for suspected IBS in primary care. Across six European countries, 5-68\% of PCPs surveyed said they would employ faecal occult blood tests, 50$75 \%$ would request an erythrocyte sedimentation rate test and $5-67 \%$ would use colonoscopy (2008). ${ }^{19}$ In the US,
$74 \%$ of PCPs surveyed said they would use faecal occult blood tests, $48 \%$ would request erythrocyte sedimentation rate tests and $17 \%$ would test for coeliac disease markers (2006). ${ }^{23}$ Other tests reported to be commonly used by US PCPs in this study were complete blood count (74\% of PCPs), electrolyte (61\%), liver (56\%) and thyroid function tests (36\%). ${ }^{23}$ Figure 2 shows the extreme variation in the types of diagnostic tests performed in patients with IBS, based on database records, ${ }^{21,24,25}$ and prospective questionnaires ${ }^{22}$ and interviews. ${ }^{20}$ Other common tests not shown in Figure 2 included those for coeliac disease $(16 \%),{ }^{24}$ C-reactive protein $(27 \%),{ }^{24}$ and thyroid function $\left(15 \%{ }^{24}\right.$ and $\left.36 \%{ }^{22}\right)$.

\section{Factors influencing diagnostic approach}

The age of both the patient and the PCP appears to have a significant impact on the diagnostic approach to IBS. Yawn et al. (2001) found that US patients who were over 50 years of age had colon imaging tests nearly twice as

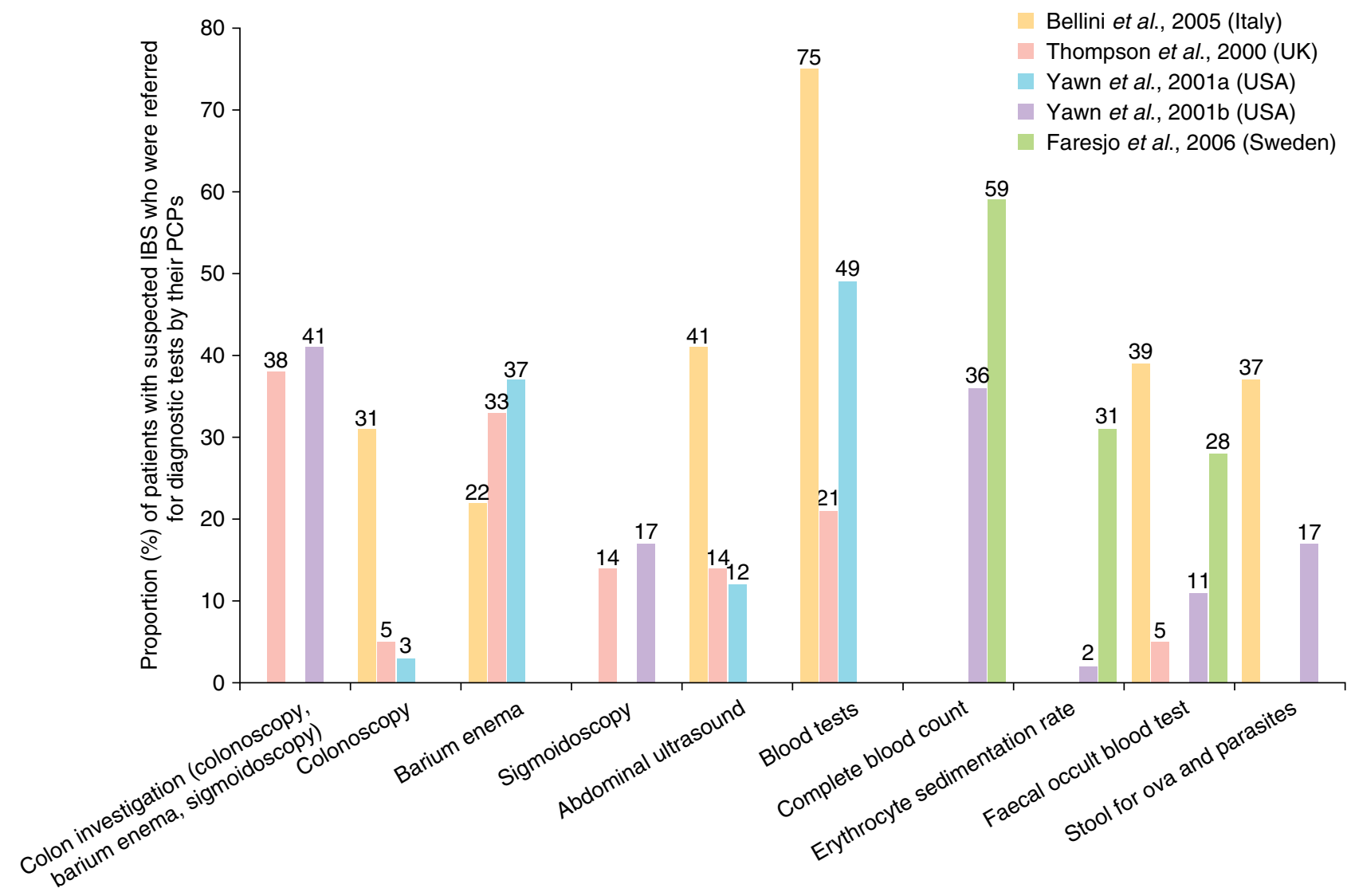

Figure 2 | Diagnostic tests ordered for patients with irritable bowel syndrome by their primary care physician. Only diagnostic tests for which data were available in at least two studies are included. 
often as patients under 50 years of age (based on medical chart review: $74 \%$ vs. $38 \%$ ), an age cut-off often recommended for this diagnostic test. ${ }^{25}$ Similarly, $14 \%$ of patients under 45 years of age received colon investigations compared with $58 \%$ of patients over 45 years of age in a UK study, based on prospective interviews $(2000),{ }^{20}$ and Italian PCPs in another study using prospective questionnaires (2005) ordered a barium enema more often for older ( $>50$ years) patients than for younger patients ( $\leq 50$ years) ( $35 \%$ vs. $11 \% ; P<0.001) .{ }^{22}$ In the Netherlands, Bijkerk et al. (2003) found that $48 \%$ of PCPs did not even consider diagnostic tests in patients younger than 50 years. ${ }^{26}$ Rectoscopy was more frequent in older $(>45$ years $)$ vs. younger ( $\leq 45$ years $)$ patients $(P<0.0001)$ based on medical chart review in the Swedish study by Faresjo et al. ${ }^{24}$ Laboratory tests were also more common in older patients, except for C-reactive protein and tests for coeliac disease, which were more frequent among younger patients (2006). ${ }^{24}$

Age (presumably a proxy for experience) also influenced diagnostic testing patterns in UK primary care in the study by Thompson et al. (1997), with a higher proportion of PCPs aged under (vs. over) 45 years of age saying they never or rarely use certain tests for excluding organic disease (barium enema: $65 \%$ vs. $21 \%$; sigmoidoscopy: $61 \%$ vs. $21 \%$; occult blood tests: $56 \%$ vs. $26 \%$; small bowel x-ray: $93 \%$ vs. $88 \%$; barium meal: $70 \%$ vs. $47 \%$; ultrasound: $79 \%$ vs. $54 \%{ }^{27}$ In contrast, Bellini et al. (2005) found that PCPs with more than 20 years of experience requested diagnostic tests less often than those with less experience $(20 \%$ vs. $5 \% ; P<0.001)$, based on data gathered using prospective questionnaires. ${ }^{22}$

In two studies [Italy (2005): prospective questionnaire $^{22}$ and the US (2001): medical chart review ${ }^{21}$ ] there was no difference in the frequency of requests for diagnostic tests between men and women. However, rectoscopy was more frequent among women $(P<0.005)$ than among men based on medical chart review in the study by Faresjo et al. (2006). ${ }^{24}$ Laboratory tests were more common for men than for women $(78 \%$ vs. $71 \%$; $P<0.05$, adjusted for age), with the exception of thyroid hormone tests which were more frequent among women. ${ }^{24}$

In US primary care, physicians who believed IBS to be a diagnosis of exclusion ordered 1.6 more tests on average for IBS-D patients than those who did not, and consumed \$364 more on average per patient $(2010){ }^{28}$ Similarly, in primary care in Denmark (2013), ${ }^{29}$ a strategy of exclusion for diagnosing IBS cost more per patient than a positive diagnostic strategy ( $\$ 5075$ vs. $\$ 3160$ )

\section{Knowledge of symptoms and diagnostic criteria}

Primary care physicians appear to be guided by key symptoms of IBS (abdominal pain, altered bowel habits and bloating). In a study conducted in Saudi Arabia (2012), 97\% of PCPs recognised abdominal pain as a symptom of IBS, followed by $83 \%$ for altered bowel habit and $77 \%$ for bloating. ${ }^{30}$ Bijkerk et al. (2003) found that $63 \%$ of PCPs in the Netherlands considered recurrent abdominal pain lasting more than 3 months as crucial for diagnosing IBS. ${ }^{26}$ PCPs in the UK and the Netherlands (2009) defined IBS as a combination of symptoms with no explained organic cause, focusing on changed defaecation pattern and abdominal pain. ${ }^{31}$ Similarly, Bellini et al. (2005) found that PCPs in Italy considered the most important symptoms for diagnosing IBS to be changes in bowel habits (96\%), abdominal pain/discomfort relieved by evacuation (82\%) and abdominal bloating (79\%). ${ }^{22}$ Nearly half of US PCPs could identify typical IBS symptoms in another study (2003). ${ }^{32}$ Across nine studies, few PCPs (2-36\%, median 20.5) had heard of formal criteria for IBS (Rome I, Rome II or Manning; Figure 3a). ${ }^{19}, 22,26,27,32-36$

\section{Use of diagnostic criteria}

The proportion of PCPs saying that they used formal diagnostic criteria to diagnose IBS was low across six studies (Figure 3b), ${ }^{19}, 22,27,33-35$ with one exception from a survey of Romanian PCPs (2006) in which 99\% stated that they used Rome II diagnostic criteria for IBS; the participants had recently attended courses on IBS and functional bowel disorders (study not included in Figure 3).

Five European studies assessed the proportion of IBS diagnoses made by PCPs that also met Manning or Rome criteria for IBS (Table 1). ${ }^{20,26,37-39}$ The highest specificity was observed for Rome III criteria in a study conducted in Denmark by Engsbro et al. (2013), with $75 \%$ of 499 patients diagnosed with IBS by their PCP meeting these criteria. ${ }^{38}$ However, the methodology used in this article may have biased towards a high specificity for Rome III criteria. PCPs who participated were asked to recruit all patients aged 18-50 years who they considered to have IBS. While not formally provided with information about diagnostic criteria by the investigators, it is hard to imagine that PCPs would not seek out criteria for IBS upon entry into such a study. Furthermore, PCPs who are already confident in diagnosing IBS may be more inclined towards participation than those who are not. Interestingly, the lowest specificity for IBS diagnostic criteria was also reported for the Rome III criteria 
A. P. S. Hungin et al.
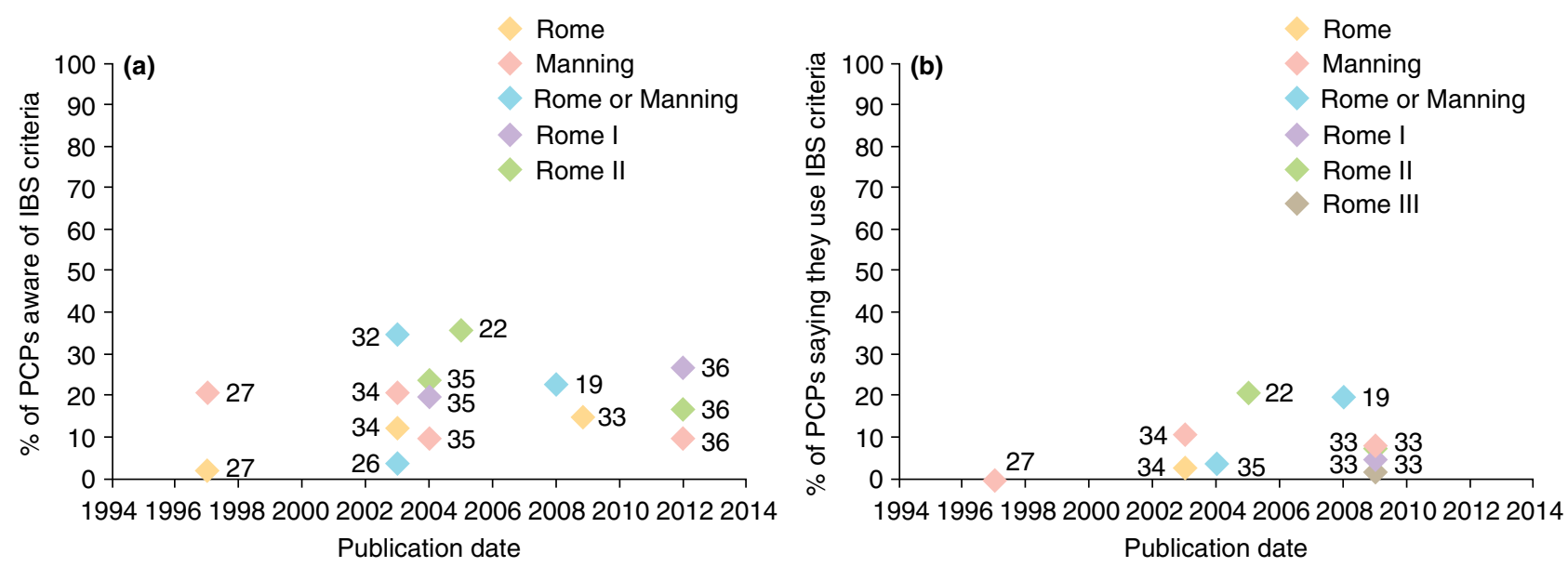

Figure 3 | (a) Awareness and (b) use of formal diagnostic criteria for IBS. Reference numbers of the corresponding studies are shown beside data points.

$(24 \%){ }^{37}$ The data-collection period for this study occurred before Rome III criteria were published, and may thus provide a truer reflection (though only in one country) of the extent to which formal diagnostic criteria for IBS line up with how PCPs tend to diagnose this disease.

The sensitivity of the Rome II criteria was low (18$39 \%)$ in the three studies reporting these data. ${ }^{26,37,39}$ In two studies, $20 \%$ (Norway) $^{39}$ and $0 \%$ (Thailand) $^{40}$ of patients meeting Rome II criteria and Rome III criteria, respectively, were subsequently diagnosed with IBS by their PCP.

\section{Diagnostic confidence}

Several lines of evidence suggest that most PCPs consider IBS to be a diagnosis of exclusion (i.e. organic causes should be excluded before diagnosing IBS). These include: structured interviews where this view was given by PCPs (2009 and 2013), ${ }^{31,41}$ including one study (2010) in which $72 \%$ of PCPs expressed this view; ${ }^{28}$ the high proportion of PCPs across studies (49-65\%; $1997-$ 2006) that rank the exclusion of organic disease as their primary concern; ${ }^{23}, 26,27$ and the high frequency of diagnostic testing among PCPs (see previous section 'Use of diagnostic tests'). ${ }^{19-21}$

Despite the prevalent belief among PCPs that IBS is a diagnosis of exclusion they are largely confident that they can make a diagnosis of IBS themselves. In the US study by Longstreth et al. (2003), PCPs ranked IBS as fourth behind heartburn, back pain and headache in terms of diagnostic confidence, ${ }^{32}$ while UK PCPs did not regard IBS as more difficult to distinguish from organic

Table 1| Overlap between primary care physician (PCP)- and criteria-based diagnosis of irritable bowel syndrome (IBS)

\begin{tabular}{|c|c|c|c|c|c|c|}
\hline & Country & $\begin{array}{c}\text { Dates } \\
\text { conducted }\end{array}$ & $\begin{array}{l}\text { Manning } \\
\text { (1978) }\end{array}$ & $\begin{array}{c}\text { Rome I (1992) } \\
\text { or Manning (1978) }\end{array}$ & $\begin{array}{l}\text { Rome II } \\
\text { (1996) }\end{array}$ & $\begin{array}{l}\text { Rome III } \\
\text { (2006) }\end{array}$ \\
\hline \multicolumn{7}{|c|}{$\%$ of patients with PCP IBS diagnosis who met IBS criteria } \\
\hline Bijkerk et al., 2003 & The Netherlands & NS & 62 & - & 18 & - \\
\hline Thompson et al., 2000 & UK & 1995 & - & 82 & - & - \\
\hline Anastasiou et al., 2008 & Greece & 1996-2000 & 69 & - & 32 & 24 \\
\hline Vandvik et al., 2004 & Norway & 2001 & - & - & 39 & - \\
\hline Engsbro et al., 2013 & Denmark & $2008-2010$ & - & - & - & 75 \\
\hline \multicolumn{7}{|c|}{$\%$ of patients meeting IBS criteria who were diagnosed with IBS by PCP } \\
\hline Thompson et al., 2000 & UK & 1995 & - & 58 & - & - \\
\hline Vandvik et al., 2004 & Norway & 2001 & - & - & 20 & - \\
\hline $\begin{array}{l}\text { Thanapirom et al. } 2012 \\
\text { (abstract) }\end{array}$ & Thailand & 2012 & - & - & - & 0 \\
\hline
\end{tabular}

NS, not specified. 
disease than pelvic pain, headache or backache in the study by Thompson et al. (1997). ${ }^{27}$ In the latter study, $37 \%$ of PCPs felt able to diagnose IBS over $50 \%$ of the time at the initial visit without further testing. In the study by Lacy et al., 53\% of PCPs in the US felt 'very comfortable' making a new IBS diagnosis at the initial visit in the absence of alarm signs. ${ }^{23}$

\section{Referral rates}

In the European survey by Seifert et al. (2008), the proportion of PCPs who would seek specialist referral before making a diagnosis of IBS was $7 \%$ in the Netherlands, $10-15 \%$ in England, $15-20 \%$ in Spain, and $25-32 \%$ for Greece, Poland and the Czech Republic. ${ }^{19}$

The proportion of patients with IBS referred by their PCP to a gastroenterologist was similar based on prospective questionnaires in two European studies reporting data $\left[20 \%(2000)^{20}\right.$ and $\left.23 \%(2005)^{22}\right]$, but lower in one US study $[4 \%(2001)] .^{25}$ In Saudi Arabia, $40 \%$ of PCPs surveyed said that they would eventually refer an IBS case to a gastroenterologist. ${ }^{30}$ The proportion of PCPs referring patients with IBS to a mental health provider was similar in two studies using prospective questionnaires that reported these data $\left[9 \%(2004)^{42}\right.$ and $12 \%(2005)^{22}$ ]. Referral to a dietician was also common in these studies $\left(7 \%^{22}\right.$ and $8 \%{ }^{42}$ ) as was referral to a gynaecologist in one of the studies (19\%). ${ }^{22}$

\section{Factors influencing referral}

A variety of reasons for referral of patients with IBS by PCPs were reported across studies. In one US $(2006)^{23}$ and two European studies [Germany (2009) ${ }^{33}$ and the UK $\left.(1997)^{27}\right], \quad 18-64 \%$ of patients were referred because of an unclear diagnosis and 24-54\% were referred owing to insufficient therapeutic response or patient dissatisfaction. Less than a quarter of the US patients $(2006)^{23}$ and $16 \%$ of Italian patients in another study $(2006)^{43}$ were referred to a specialist because they needed reassurance, while $3 \%$ and $34 \%$ of referrals were at the request of the patient in the studies conducted in Germany $(2009)^{33}$ and Italy (2006), ${ }^{43}$ respectively. The most common reason for referral for the US patients was the presence of alarm features, while the three most common reasons for referral to a psychiatrist were co-existing anxiety or depression, history of physical or sexual abuse and symptoms refractory to therapy. ${ }^{23}$ Denial of a role for stress in IBS was a significant predictor of referral to a specialist by UK PCPs after logistic regression, as was multiple diagnostic testing and the presence of frequent bowel movements, in a study by Thompson et al. (2000). ${ }^{20}$

Thompson et al. (1997) found a higher proportion of male doctors than female doctors said they referred their patients (18\% vs. $7 \%$ ) based on retrospective questionnaires administered in the $\mathrm{UK}$, and referral rates were also higher for older doctors (43-60 years old) compared with younger doctors (31-42 years old) in this study (19\% vs. $10 \%) .{ }^{27}$ PCPs' decisions regarding referrals did not seem to vary with clinical presentation or patient age in Italian patients (2005), ${ }^{22}$ or in relation to IBS subtype in US patients (2006), ${ }^{23}$ according to prospectively applied questionnaires.

\section{Views on aetiology and pathophysiology}

Most PCPs recognise that psychological comorbidities are common in IBS but opinions vary about their aetiological significance. Across three studies [Saudi Arabia (2012), the Netherlands (2003) and the US (2006)], 55$71 \%$ of PCPs identified stress, anxiety (or 'nervousness') and depression as being associated with symptoms of IBS, ${ }^{23,} 26,30$ while $45 \%$ of UK PCPs agreed that IBS is a nervous complaint in another study (2004). ${ }^{44}$ Casiday et al. (2009) reported that PCPs frequently saw IBS as a consequence of disordered bowel activity in response to stress. ${ }^{31}$

Two studies, which between them covered seven European countries [Czech Republic, Greece, the Netherlands, Poland, UK and Spain (2008), ${ }^{19}$ and Germany (2009) ${ }^{33}$ ], reported that about a quarter to two-thirds of PCPs believed IBS had a psychiatric or psychological component, while none thought so in another study in Romania (2006). ${ }^{45}$ In other studies, $49 \%$ of UK PCPs thought IBS was psychosomatic (2003), ${ }^{34}$ while psychological and psychiatric factors were ranked second by Italian PCPs (after intestinal motility disorder) as the most probable cause of IBS symptoms (2005). ${ }^{22}$ Another study in UK primary care by Thompson et al. (1997) found that $87 \%$ of PCPs thought IBS aetiology was sometimes physical and sometimes psychological; however, only $14 \%$ and $7 \%$ would apply a psychological or psychiatric label, respectively. ${ }^{27}$

There are also differences in PCPs' views of the aetiological significance of visceral pain sensitivity, motility, enteric infection and sexual abuse. Gut hypersensitivity was believed to be an aetiological factor for IBS by $26 \%$ of Dutch PCPs $(2003)^{26}$ and $54 \%$ of US PCPs (2006). ${ }^{23}$ The latter study also found that most PCPs believed IBS was a disorder of both gut hypersensitivity and gastrointestinal motility. ${ }^{23}$ The proportion of PCPs who consid- 
ered IBS to be a motility disorder ranged from $2 \%$ in Romania (2006), ${ }^{45}$ to $62 \%$ in the US (2006), ${ }^{23}$ and $49 \%$ and $62 \%$ in Germany (2009) $)^{33}$ and the Netherlands (2003), ${ }^{26}$ respectively. In one study (2009), ${ }^{31}$ Dutch PCPs considered smoking, caffeine, diet, 'hasty lifestyle' and lack of exercise as other possible triggers of IBS symptoms, while UK PCPs considered food, infection and travel as other possible triggers. ${ }^{31}$ The proportion of PCPs who believe infection or food intolerance causes IBS was low $(<5 \%)$ in Saudi Arabia $(2012)^{30}$ and the Netherlands $(2003){ }^{26}$

\section{Views on IBS management}

Relief of symptoms was rated by US PCPs as their second greatest concern behind excluding organic disease, with only $22 \%$ rating this as their main objective (2006). ${ }^{23}$ In contrast, $73 \%$ believed that symptom relief was the patients' primary concern.

Despite widely held beliefs that IBS has a psychological component [three UK studies (1997-2013); one European study (six countries; 2008); one Italian study (2005) and one German study (2009)], ${ }^{19}, 22,27,33,34,41$ PCPs are often reluctant to consider mental health interventions (2013 and 2004; UK). ${ }^{41,46}$ Reasons for this include a lack of familiarity with such interventions, perceived patient resistance to psychological treatment and doubts of the strength of evidence for psychological intervention (2004; UK), ${ }^{46}$ as well as the belief that the condition can be managed effectively and adequately in primary care (2013 and 2004; UK). ${ }^{41,46}$

In terms of perspectives on the effectiveness of IBS treatment, Cox et al. (2004) found that $40 \%$ of PCPs in the UK study agreed that IBS responded mainly to the placebo effect of personal care and attention, and most (61\%) were unsure about or disagreed with the statement that IBS symptoms mainly respond to medical therapy. ${ }^{44}$ Most PCPs were also unsure about or disagreed with the statements that existing treatment regimens $(54 \%)$ or dietary advice $(59 \%)$ are effective. ${ }^{44}$ In the same study, $73 \%$ and $77 \%$ of PCPs agreed that hypnotherapy could help patients with physical and psychological problems, respectively. ${ }^{44}$

\section{Management approaches}

UK PCPs in the study by Casiday et al. (2009) stated that their main focus was managing symptoms and reassuring patients with IBS. $^{31}$ In terms of management goals for pharmacotherapy, Bijkerk et al. (2003) found that $70 \%$ of Dutch PCPs considered global symptom improvement to be their main aim, while $28 \%$ aimed mainly to improve predominant IBS symptoms and $2 \%$ aimed mainly to improve quality of life. ${ }^{26}$ In this study, 93\% of respondents said they provided dietary advice to their patients, $77 \%$ used counselling, $63 \%$ gave routine lifestyle advice, $55 \%$ prescribed drug therapy and $4 \%$ provided behavioural therapy. ${ }^{26}$ A similarly high proportion of 70 PCPs surveyed in the Icelandic study by Olafstdottir et al. (2012) said they provided dietary advice (98\%) and education about IBS (90\%); advice around relaxation and exercise was less common $(\sim 15 \%) .{ }^{36}$ Counselling and patient education were only provided for $18 \%$ of patients with IBS in the 3 years after diagnosis in one US study (2001). ${ }^{21}$ In another study in US primary care (2004) that used prospective questionnaires, 55\% of patients with IBS received education about the cause of their symptoms, $63 \%$ received dietary advice, 50\% exercise advise and 37\% lifestyle advice on how to reduce stress. ${ }^{42}$

Among German PCPs, 96\% of those surveyed stated that they prescribed drug therapy, while psychotherapy and alternative therapies (such as homoeopathy, acupuncture, phytotherapy dietary therapy or probiotics) were recommended by $55 \%$ and $61 \%$ of PCPs, respectively (2009). ${ }^{33}$ Only $8 \%$ of US primary care patients with IBS were referred to naturopaths in the study by Whitehead et al. (2004), ${ }^{42}$ based on prospective questionnaires.

Most UK and Dutch PCPs surveyed by Casiday et al. (2009) said they prescribed fibre for IBS. ${ }^{31}$ The UK PCPs said they readily prescribed medications, while the Dutch PCPs preferred not to prescribe any drugs, unless requested by the patient, based on a belief that limited evidence for efficacy exists. ${ }^{31}$

Figure 4 shows the large variation in the types of medications used to treat IBS in primary care in terms of the proportion of PCPs prescribing based on retrospective questionnaires, ${ }^{26,} 30,36$ while Figure 5 shows the actual medications received by patients based on database records, ${ }^{21,24}$ prospective $^{22}$ and retrospective ${ }^{42,47}$ questionnaires.

\section{Factors influencing management approaches}

In the Swedish study by Faresjo et al. (2006), all prescriptions for IBS increased with increasing age except for anti-diarrhoeal agents, which were more common among younger patients based on medical chart review. ${ }^{24}$ Prescription of anti-depressants was independently associated with being female $(P<0.03) .{ }^{24}$ In the only other study reporting such data (2001), which also used data from medical charts, women in the US with IBS were 
slightly more likely to use medications for bowel dysfunction (laxatives and antidiarrhoeals) than men $(P=0.05)$, and men were more likely to have prescriptions for histamine blockers $(P=0.01)$, anti-depressants and anti-anxiety medications $(P=0.03){ }^{21}$

\section{DISCUSSION}

Most PCPs consider IBS to be a diagnosis of exclusion, but one that can be reached in primary care. There has been much emphasis recently about the desirability of an initial positive diagnosis. While it appears that most PCPs do make a tentative IBS diagnosis from the start, they still tend to use additional testing to confirm it.

To our knowledge, this is the first systematic review of published studies providing insight into the perceptions, diagnosis and management of IBS, specifically from a primary care perspective. The main limitation of this review relates to the large variety of data reported, which make it difficult to draw clearly defined conclusions. Another limitation is that only broad pre-defined selection criteria could be applied owing to the wide scope of the study question. Furthermore, like any review seeking to capture current thinking in an ever-evolving field, it is inevitable that some of the data presented here no longer represent current practice and that newer trends have not yet made it into the literature. In terms of the quality of the included studies, some were based on retrospectively applied questionnaires, which are prone to recall bias, although many studies, including those reporting diagnostic tests used and medications prescribed in patients with IBS, used more reliable methods such as prospective questionnaires and interviews, and medical records. Despite the limitations, this is the most comprehensive assessment yet of this topic and we believe some useful, though tentative, inferences can be drawn.

We have attempted to synthesise papers from across countries - this strengthens the article in scope but reduces the generalisability of conclusions because of locally prevailing factors. In particular, differences in healthcare systems across countries will have a bearing on the way patients are diagnosed and managed further. In settings where there is a clear delineation between primary and secondary care referral is likely to be restricted to patients whose diagnosis is uncertain or to situations where factors such as patient reticence drive towards a specialist opinion or colonoscopy. For example, in the $\mathrm{UK}$, economic pressures to reduce specialist referrals and colonoscopy mean that primary care management of IBS predominates. In countries with a mixed system, whereby specialists work in the community, barriers to specialist investigations may be reduced and the prevalence of such diagnostic strategies is thus likely to be higher. Factors underlying which diagnostic strategies predominate in different countries include the type of reimbursement system, the level of easy (and low cost) access to investigational services and how the consulting clinician is incentivised. These differences may even be localised within countries, for example between those patients who are privately insured and those entirely within the state system.

Primary care physicians have a heterogenous view of the causes of IBS. Their perceptions of the factors associated with IBS were related to the presence of stress and nervousness, with some indicating that gut hypersensitivity plays a role, and a very small fraction identifying food allergies as a factor. No obvious single explanatory model for the symptoms of IBS itself was discernible, which is probably why treatment approaches were found to vary so greatly. Relatively few PCPs had heard of Manning or Rome diagnostic criteria, and still fewer used them in their practice, though recent studies assessing the awareness or use of Rome III criteria were lacking. Despite a lack of awareness and use of formal diagnostic criteria for IBS, most PCPs could identify typical symptoms of IBS.

Contrary to the views of many outside primary care, the literature indicated that PCPs do not, in general, make an immediate, positive diagnosis of IBS but access tests, including colonoscopy, to exclude other problems. This follows the necessarily global approach that PCPs

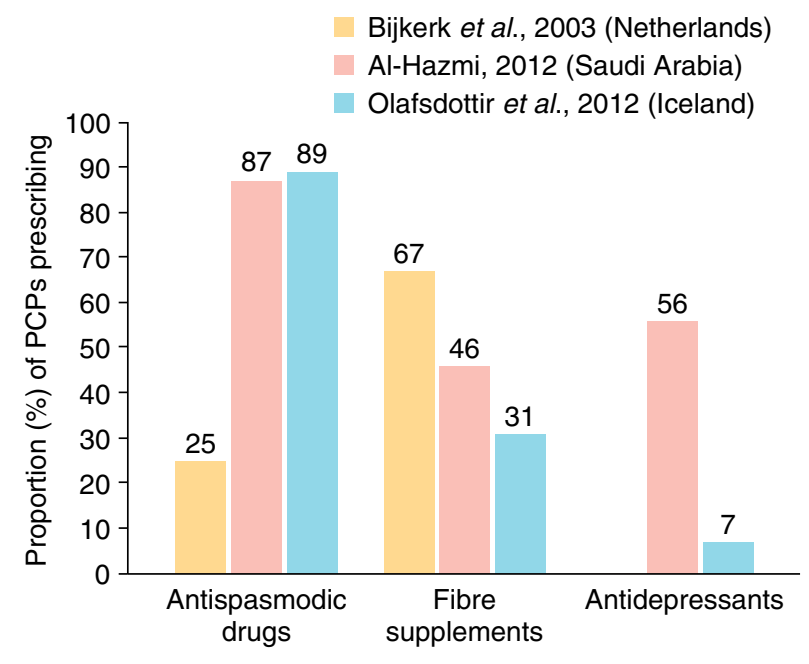

Figure 4 | Proportion of primary care physicians prescribing medications for patients with irritable bowel syndrome. Only medications for which data were available in at least two studies are included. 
A. P. S. Hungin et al.
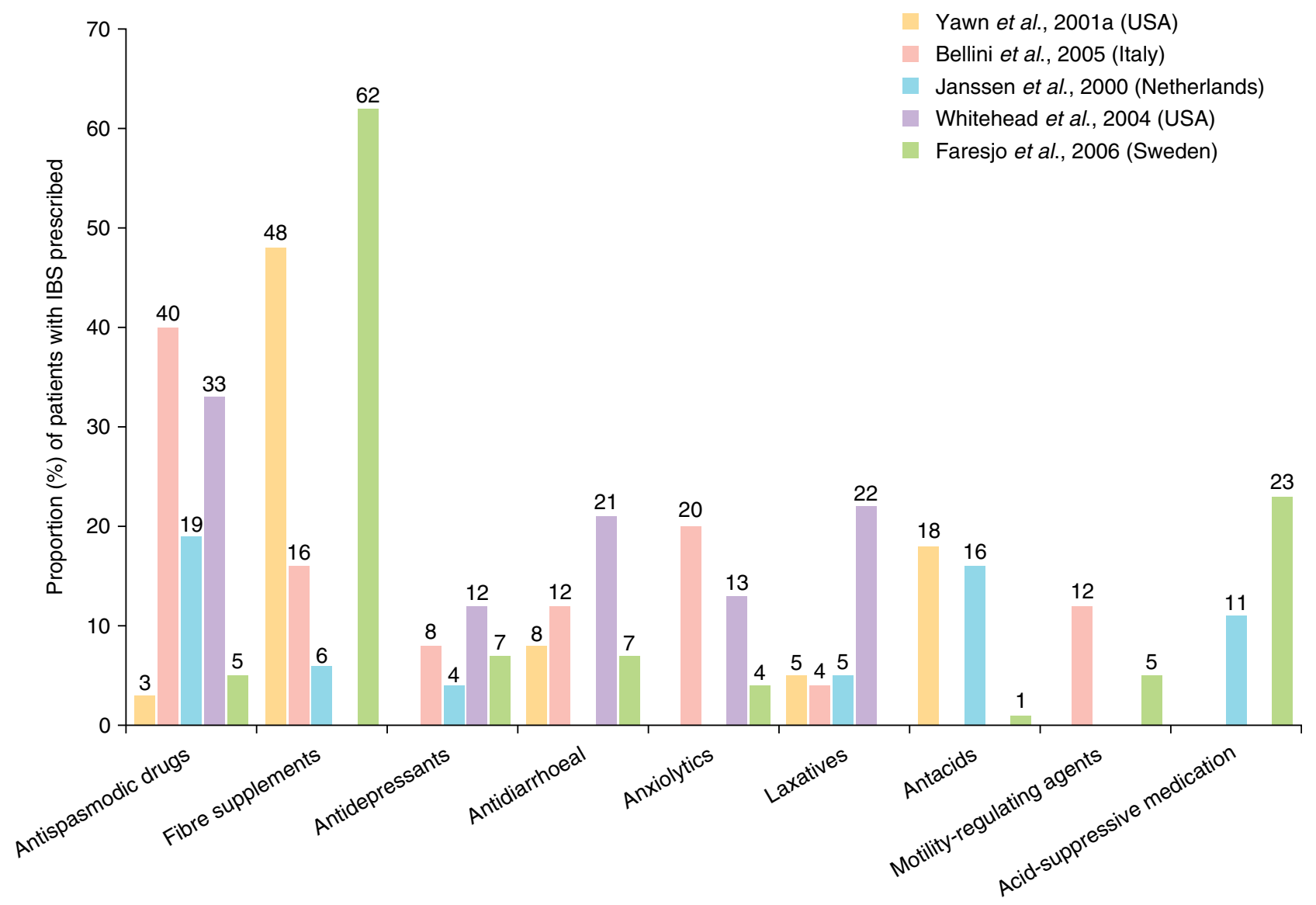

Figure 5 | Proportion of patients with irritable bowel syndrome being prescribed medications by their primary care physician. Only medications for which data were available in at least two studies are included.

need to take in patients presenting with heterogeneous symptoms from different possible causes. An example is cough, which in the vast majority of cases is a simple, self-limiting problem, but for which a lack of vigilance may result in a serious lesion being missed. An equivalent situation exists for IBS and for many PCPs this means they will test early. In this study diagnostic management in IBS varied largely between different healthcare settings, resulting in a wide variation in confidence about diagnosing IBS. Diagnostic testing by PCPs was very common overall, though the types of tests used varied greatly. Taking the above factors into consideration, in combination with the low proportion of PCPs who would seek a specialist referral before making a diagnosis of IBS (7-32\%), ${ }^{19}$ it appears that most PCPs consider IBS to be a diagnosis of exclusion, but one that can be reached in primary care.

The use of multiple diagnostic tests to exclude IBS and uncertainty around treatment approaches seems to be reflected by patients' experience of primary care, which can leave some feeling confused and frustrated. $^{48,49}$ Only a small proportion of patients with IBS $(4-23 \%)^{20,22,25}$ are referred to secondary care. Reasons for referral include an unclear diagnosis, insufficient therapeutic response (which, ironically is a common factor in IBS and not necessarily bettered in secondary care) and patient dissatisfaction. Primary care physicians lack consensus on the best conceptual model for understanding IBS: they exhibit regional differences in their beliefs about aetiology, diagnosis, the role of psychological factors and treatment guidelines. Although much variation exists between PCPs in different settings in terms of their diagnostic and management behaviour, the diagnosis of IBS is mainly based on excluding other problems, even if a tentative diagnosis is made early. Diagnostic criteria, mainly established from secondary care, are largely unknown or not applied in primary care, at least according to the most recent literature. Stress and other psychological factors are considered an important part of IBS in primary care. 
Combined with perceived uncertainties and the stress of getting things wrong the PCP is often in a difficult position. It is important to appreciate that uncertainty and a lack of confidence in a symptom-based positive diagnosis of IBS greatly influences clinical behaviour, particularly when progress is not made with the patient and available options are rapidly exhausted. It is therefore not surprising that many PCPs adapt their practice towards early investigation. While a positive diagnosis of IBS by PCPs in low-risk patients would be less costly than a diagnosis of exclusion, this is not the way most PCPs practice. Furthermore, there is evidence that none of the formal diagnostic criteria currently available sufficiently, conclusively distinguish IBS from organic diseases. ${ }^{50}$ Until this changes, bowel investigations, such as colonoscopy, will continue to be important for PCPs to exclude organic disease and, paradoxically, there may be calls for this to be made more easily available; often differences in diagnostic strategies are related to health care systems and their limitations. The ongoing development of new diagnostic algorithms, based on symptoms, comorbidity and psychosocial profile, in combination with new biomarker point-of-care tests, such as for calprotectin, should facilitate and support more efficient discrimination between organic and functional bowel disease by PCPs and reduce the use of lower GI endoscopy. Further educational initiatives alone and dissemination of diagnostic criteria may be insufficient - while an early, positive diagnosis of IBS in primary care is likely to be accurate and efficient there are many barriers before this is accepted in pragmatic practice. Specialists, too, will need to align with this ethic if it is not to be undermined.

\section{AUTHORSHIP}

Guarantor of the article: A Pali S. Hungin.

Author contributions: A Pali S Hungin took a lead in determining the study concept and design, interpreting the data and critically reviewing the manuscript for important clinical and intellectual content. Michael Molloy-Bland performed the literature searches and played a major role in drafting and critically reviewing the manuscript for important intellectual content. Richard Claes independently verified the literature searches and data extraction and critically reviewed the manuscript for important intellectual content. Joel Heidelbaugh, William Cayley, Jean Muris, Bohumil Seifert, Greg Rubin and Niek de Wit played major roles in interpreting the data and critically reviewing the manuscript for important clinical and intellectual content. All authors have approved the final draft of the manuscript, including the authorship list.

\section{ACKNOWLEDGEMENTS}

We thank the members of the Rome Foundation Committee for their support and valuable intellectual contributions to this manuscript.

Declaration of personal interests: A Pali S Hungin has been an advisory board member and speaker for Reckitt Benckiser, Danone and Novartis. Greg Rubin has provided consultancy advice and has developed educational materials for Almirall. Michael Molloy-Bland and Richard Claes are employed as Medical Writers at Oxford PharmaGenesis ${ }^{\mathrm{TM}}$ Ltd, Oxford, UK, which collaborated on this project with the School of Medicine, Pharmacy and Health, Durham University, Durham, UK. William Cayley, Joel Heidelbaugh, Jean Muris, Bohumil Seifert and Niek de Wit have no personal interests to declare.

Declaration of funding interests: Michael Molloy-Bland and Richard Claes are employees of Oxford PharmaGenesis $^{\text {TM }}$ Ltd, Oxford, UK, which received a departmental grant for this project from the School of Medicine, Pharmacy and Health, Durham University, Durham, UK.

\section{SUPPORTING INFORMATION}

Additional Supporting Information may be found in the online version of this article:

Table S1. Characteristics of the included studies.

\section{REFERENCES}

1. Fass R, Longstreth GF, Pimentel M, et al. Evidence- and consensusbased practice guidelines for the diagnosis of irritable bowel syndrome. Arch Intern Med 2001; 161: 2081-8.

2. Drossman DA, Camilleri M, Mayer EA, Whitehead WE. AGA technical review on irritable bowel syndrome. Gastroenterology 2002; 123: 2108-31.

3. Hungin AP, Chang L, Locke GR, Dennis EH, Barghout V. Irritable bowel syndrome in the United States: prevalence, symptom patterns and impact. Aliment Pharmacol Ther 2005; 21: $1365-75$.
4. Quigley EM, Abdel-Hamid H, Barbara G, et al. A global perspective on irritable bowel syndrome: a consensus statement of the World Gastroenterology Organisation Summit Task Force on irritable bowel syndrome. J Clin Gastroenterol 2012; 46: 356-66. 
5. Hungin AP, Whorwell PJ, Tack J, Mearin F. The prevalence, patterns and impact of irritable bowel syndrome: an international survey of 40,000 subjects. Aliment Pharmacol Ther 2003; 17: 64350.

6. Gwee KA, Lu CL, Ghoshal UC. Epidemiology of irritable bowel syndrome in Asia: something old, something new, something borrowed. $J$ Gastroenterol Hepatol 2009; 24: 1601-7.

7. Longstreth GF, Thompson WG, Chey WD, Houghton LA, Mearin F, Spiller RC. Functional bowel disorders. Gastroenterology 2006; 130: 1480-91.

8. Manning AP, Thompson WG, Heaton KW, Morris AF. Towards positive diagnosis of the irritable bowel. $\mathrm{Br}$ Med J 1978; 2: 653-4.

9. Rubin G, De Wit N, Meineche-Schmidt V, Seifert B, Hall N, Hungin P. The diagnosis of IBS in primary care: consensus development using nominal group technique. Fam Pract 2006; 23: 687-92.

10. Dang J, Ardila-Hani A, Amichai MM, Chua K, Pimentel M. Systematic review of diagnostic criteria for IBS demonstrates poor validity and utilization of Rome III. Neurogastroenterol Motil 2012; 24: 853-e397.

11. Whitehead WE, Drossman DA. Validation of symptom-based diagnostic criteria for irritable bowel syndrome: a critical review. Am J Gastroenterol 2010; 105: 814-20.

12. Suares NC, Ford AC. Diagnosis and treatment of irritable bowel syndrome. Discov Med 2011; 11: 425-33.

13. Ruepert L, Quartero AO, de Wit NJ, van der Heijden GJ, Rubin G, Muris JW. Bulking agents, antispasmodics and antidepressants for the treatment of irritable bowel syndrome. Cochrane Database Syst Rev 2011; (8): CD003460.

14. Zijdenbos IL, de Wit NJ, van der Heijden GJ, Rubin G, Quartero AO. Psychological treatments for the management of IBS. Cochrane Database Syst Rev 2009; 1: CD006442.

15. Hungin AP, Mulligan C, Pot B, et al. Systematic review: probiotics in the management of lower gastrointestinal symptoms in clinical practice - an evidence-based international guide. Aliment Pharmacol Ther 2013; 38: 864-86.

16. Kettell J, Jones R, Lydeard S. Reasons for consultation in irritable bowel syndrome: symptoms and patient characteristics. Br J Gen Pract 1992; 42: 459-61.

17. Dhaliwal SK, Hunt RH. Doctor-patient interaction for irritable bowel syndrome in primary care: a systematic perspective. Eur J Gastroenterol Hepatol 2004; 16: 1161-6.
18. Oberndorff-Klein Woolthuis AH, Brummer RJ, deWit NJ, Muris JW, Stockbrugger RW. Irritable bowel syndrome in general practice: an overview. Scand J Gastroenterol Suppl 2004; (241): 17-22.

19. Seifert B, Rubin G, de Wit N, et al. The management of common gastrointestinal disorders in general practice A survey by the European Society for Primary Care Gastroenterology (ESPCG) in six European countries. Dig Liver Dis 2008; 40: 659-66.

20. Thompson WG, Heaton KW, Smyth GT, Smyth C. Irritable bowel syndrome in general practice: prevalence, characteristics, and referral. Gut 2000; 46: 78-82.

21. Yawn BP, Locke GR 3rd, Lydick E, Wollan PC, Bertram SL, Kurland MJ. Diagnosis and care of irritable bowel syndrome in a community-based population. Am J Manag Care 2001; 7: 585-92.

22. Bellini M, Tosetti C, Costa F, et al. The general practitioner's approach to irritable bowel syndrome: from intention to practice. Dig Liver Dis 2005; 37: 934-9.

23. Lacy BE, Rosemore J, Robertson D, Corbin DA, Grau M, Crowell MD. Physicians' attitudes and practices in the evaluation and treatment of irritable bowel syndrome. Scand J Gastroenterol 2006; 41: 892-902.

24. Faresjo A, Grodzinsky E, Foldevi M, Johansson S, Wallander MA. Patients with irritable bowel syndrome in primary care appear not to be heavy healthcare utilizers. Aliment Pharmacol Ther 2006; 23: 807-14.

25. Yawn BP, Lydick E, Locke GR, Wollan PC, Bertram SL, Kurland MJ. Do published guidelines for evaluation of irritable bowel syndrome reflect practice? BMC Gastroenterol 2001; 1: 11.

26. Bijkerk CJ, de Wit NJ, Stalman WA, Knottnerus JA, Hoes AW, Muris JW. Irritable bowel syndrome in primary care: the patients' and doctors' views on symptoms, etiology and management. Can J Gastroenterol 2003; 17: 363-8.

27. Thompson WG, Heaton KW, Smyth GT, Smyth C. Irritable bowel syndrome: the view from general practice. Eur J Gastroenterol Hepatol 1997; 9: 689-92.

28. Spiegel BM, Farid M, Esrailian E, Talley J, Chang L. Is irritable bowel syndrome a diagnosis of exclusion?: a survey of primary care providers, gastroenterologists, and IBS experts. Am J Gastroenterol 2010; 105: 848-58.

29. Begtrup LM, Engsbro AL, Kjeldsen J, et al. A positive diagnostic strategy is noninferior to a strategy of exclusion for patients with irritable bowel syndrome. Clin Gastroenterol Hepatol 2013; 11: 956-62 e1.

30. Al-Hazmi AH. Knowledge, attitudes, and practices of primary care physicians about irritable bowel syndrome in Northern Saudi Arabia. Saudi J Gastroenterol 2012; 18: 173-81.

31. Casiday RE, Hungin AP, Cornford CS, de Wit NJ, Blell MT. GPs' explanatory models for irritable bowel syndrome: a mismatch with patient models? Fam Pract 2009; 26: 34-9.

32. Longstreth GF, Burchette RJ. Family practitioners' attitudes and knowledge about irritable bowel syndrome: effect of a trial of physician education. Fam Pract 2003; 20: 670-4.

33. Franke A, Singer MV, Dumitrascu DL. How general practitioners manage patients with irritable bowel syndrome. Data from a German urban area. Rom J Intern Med 2009; 47: 47-53.

34. Gladman LM, Gorard DA. General practitioner and hospital specialist attitudes to functional gastrointestinal disorders. Aliment Pharmacol Ther 2003; 17: 651-4.

35. Lea R, Hopkins V, Hastleton J, Houghton LA, Whorwell PJ. Diagnostic criteria for irritable bowel syndrome: utility and applicability in clinical practice. Digestion 2004; 70: 210-3.

36. Olafsdottir LB, Gudjonsson $\mathrm{H}$, Jonsdottir HH, Jonsson JS, Bjornsson E, Thjodleifsson B. Irritable bowel syndrome: physicians' awareness and patients' experience. World J Gastroenterol 2012; 18: 3715-20.

37. Anastasiou F, Mouzas IA, Moschandreas J, Kouroumalis E, Lionis C. Exploring the agreement between diagnostic criteria for IBS in primary care in Greece. BMC Res Notes 2008; 1: 127.

38. Engsbro AL, Begtrup LM, Kjeldsen J, et al. Patients suspected of irritable bowel syndrome-cross-sectional study exploring the sensitivity of Rome III criteria in primary care. $A m \mathrm{~J}$ Gastroenterol 2013; 108: 972-80.

39. Vandvik PO, Aabakken L, Farup PG. Diagnosing irritable bowel syndrome: poor agreement between general practitioners and the Rome II criteria. Scand J Gastroenterol 2004; 39: 448-53.

40. Thanapirom K, Gonlachanvit S. The agreement between clinical diagnosis of functional gastrointestinal disorders by primary care physicians and the Rome III diagnostic criteria. Asian Pacific Digestive Week 2012; December; Bangkok, Thailand. J Gastroenterol Hepatol 2012; 27 (Suppl 5): 59-438.

41. Harkness EF, Harrington V, Hinder S, et al. GP perspectives of irritable bowel 
syndrome-an accepted illness, but management deviates from guidelines: a qualitative study. BMC Fam Pract 2013; 14: 92.

42. Whitehead WE, Levy RL, Von Korff M, et al. The usual medical care for irritable bowel syndrome. Aliment Pharmacol Ther 2004; 20: 1305-15.

43. Bellini M, Tosetti C, Stasi C, et al. The general practitioner's management of patients with a new diagnosis of irritable bowel syndrome. J Clin Gastroenterol 2006; 40: 87.

44. Cox S, de Lusignan S, Chan T. General practitioners believe that hypnotherapy could be a useful treatment for irritable bowel syndrome in primary care. $B M C$ Fam Pract 2004; 5: 22.
45. Dumitrascu DL, David L, Singer M. What general practitioners know about irritable bowel syndrome. Preliminary data from a Romanian province. J Gastrointestin Liver Dis 2006; 15: 227-30.

46. Raine R, Carter S, Sensky T, Black $\mathrm{N}$. General practitioners' perceptions of chronic fatigue syndrome and beliefs about its management, compared with irritable bowel syndrome: qualitative study. $B M J$ 2004; 328: 1354-7.

47. Janssen HA, Borghouts JA, Muris JW, Metsemakers JF, Koes BW, Knottnerus JA. Health status and management of chronic non-specific abdominal complaints in general practice. $\mathrm{Br} \mathrm{J} \mathrm{Gen}$ Pract 2000; 50: 375-9.
48. Casiday RE, Hungin AP, Cornford CS, de Wit NJ, Blell MT. Patients' explanatory models for irritable bowel syndrome: symptoms and treatment more important than explaining aetiology. Fam Pract 2009; 26: 40-7.

49. Meadows LM, Lackner S, Belic M. Irritable bowel syndrome. An exploration of the patient perspective. Clin Nurs Res 1997; 6: 156-70.

50. Ford AC, Bercik P, Morgan DG, Bolino C, Pintos-Sanchez MI, Moayyedi P. Validation of the Rome III criteria for the diagnosis of irritable bowel syndrome in secondary care. Gastroenterology 2013; 145: 1262-70 e1. 